\title{
New Results on Photodisintegration of ${ }^{4} \mathrm{He}$
}

\section{Tatsushi Shima*}

Research Center for Nuclear Physics, Osaka University, Ibaraki, Osaka 567-0047, Japan

E-mail: Shimalrcnp.osaka-u.ac.jp

\section{Yasuki Nagai}

Nuclear Science and Engineering Directorate, Japan Atomic Energy Agency, Tokai, Ibaraki 319-1195, Japan

E-mail: nagaiercnp.osaka-u.ac.jp

\section{Shuji Miyamoto, Sho Amano, Ken Horikawa, and Takayasu Mochizuki}

Laboratory of Advanced Science and Technology for Industry, University of Hyogo, Ako-gun, Hyogo 678-1205, Japan

E-mail: miyamotodlasti.u-hyogo.ac.jp, shodlasti.u-hyogo.ac.jp,

horiken@lasti.u-hyogo.ac.jp hochizuki@lasti.u-hyogo.ac.jp

Photonuclear reactions of ${ }^{4} \mathrm{He}$ were studied experimentally in the energy region from $27.1 \mathrm{MeV}$ to $37.2 \mathrm{MeV}$ by means of the laser Compton-backscattered $\gamma$-ray source at the NewSUBARU synchrotron radiation facility and a time-projection chamber. The absolute sensitivity of the measurement was checked with the simultaneous measurement of the photodisintegration cross section of the deuteron. The measured cross sections were found to be consistent with our previous result in the region of $29 \sim 31 \mathrm{MeV}$, while the present result on the $(\gamma, \mathrm{p})$ and $(\gamma, \mathrm{n})$ reactions suggested those cross sections become maximum at around $32 \mathrm{MeV}$, being different from the peak energy expected from the other existing data.

6th International Workshop on Chiral Dynamics, CD09

July 6-10, 2009

Bern, Switzerland

\footnotetext{
* Speaker.
} 


\section{Introduction}

Photonuclear reactions of light nuclei at low energies provide valuable information about the properties of few-nucleon systems and the details of the nucleon-nucleon interactions. Since ${ }^{4} \mathrm{He}$ has several unique features such as a self-conjugate structure and a large binding energy per nucleon like heavier nuclei, its photonuclear reactions are useful for development of sophisticated models

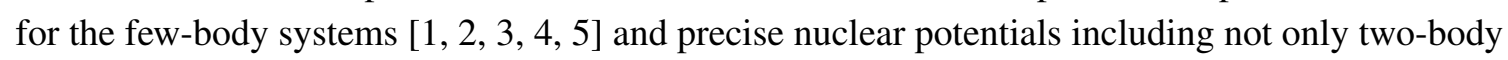
central forces but also tensor components [6], many-body forces [7, 8, 9], charge-asymmetries [10, 11], and so on. The photonuclear reactions of ${ }^{4} \mathrm{He}$ are also interesting from the astrophysical viewpoint, because they can be applied to investigate the analogous neutrino-inelastic scattering [12, 13], which is supposed to play critical roles in the neutrino-heating mechanism in Type-II supernova explosions [14, 15] and in the r-process nucleosynthesis occurring in the neutrino-driven wind [16, 17, 18]. Another recent topic related to the astrophysics is the effect of the destruction of ${ }^{4} \mathrm{He}$ by high-energy $\gamma$-rays from the decay of long-lived exotic particles as a possible solution for the problem in the abundances of the primordial lithium isotopes [19].

For the above reasons, precise data of the photonuclear reactions of ${ }^{4} \mathrm{He}$ have become highly demanded. So far, the dipole excitation of ${ }^{4} \mathrm{He}$ has been investigated through the experiments on the photodisintegration of ${ }^{4} \mathrm{He}$ as well as their inverse radiative capture reactions of ${ }^{3} \mathrm{H}(\mathrm{p}, \gamma){ }^{4} \mathrm{He}$ and ${ }^{3} \mathrm{He}(\mathrm{n}, \gamma)^{4} \mathrm{He}$ (see references in Ref. [20]). Those data, however, have suffered from serious discrepancies as large as 50 100\% below the $\gamma$-ray energy of $E_{\gamma} \sim 40 \mathrm{MeV}$, which may be caused by the influence of low-energy background $\gamma$-rays and/or uncertainties in determining experimental parameters such as geometrical acceptance of a detector, the effective thickness of the target, the absolute intensity of the incident beams, etc.

Keeping those situations in mind, we made a measurement of the photonuclear reaction cross sections of ${ }^{4} \mathrm{He}$ using the quasi-monochromatic $\gamma$-ray beam generated by the laser Compton backscattering (LCS) method at the electron storage ring TERAS in the National Institute for Advanced Industrial Science and Technology (AIST) [20]. The measured cross sections were found to monotonically increase as a function of $E_{\gamma}$ up to $\sim 30 \mathrm{MeV}$, being quite different from many previous data including the most recent result on the $(\gamma, \mathrm{n})$ cross section. In order to get more information about the excitation function of the photodisintegration of ${ }^{4} \mathrm{He}$, we made a new measurement in the extended energy region up to $E_{\gamma}=37.2 \mathrm{MeV}$ using a newly developed LCS $\gamma$-ray source at the NewSUBARU synchrotron radiation facility of the Laboratory of Advanced Science and Technology for Industry (LASTI) at the University of Hyogo, Japan.

In this paper a preliminary result of the measurement of the photonuclear reaction cross sections of ${ }^{4} \mathrm{He}$ at NewSUBARU are reported.

\section{Experimental method}

Fig. 1 shows a schematic view of the experimental setup. The NewSUBARU is a $1.5 \mathrm{GeV}$ race-track shaped electron storage ring. The LCS $\gamma$-rays were generated via the head-on collision of an electron beam and a laser light occurring in a straight section of the storage ring [21]. The $180^{\circ}$ backscattered $\gamma$-rays were extracted with a collimator made of a $10 \mathrm{~cm}$ thick lead brick, and 


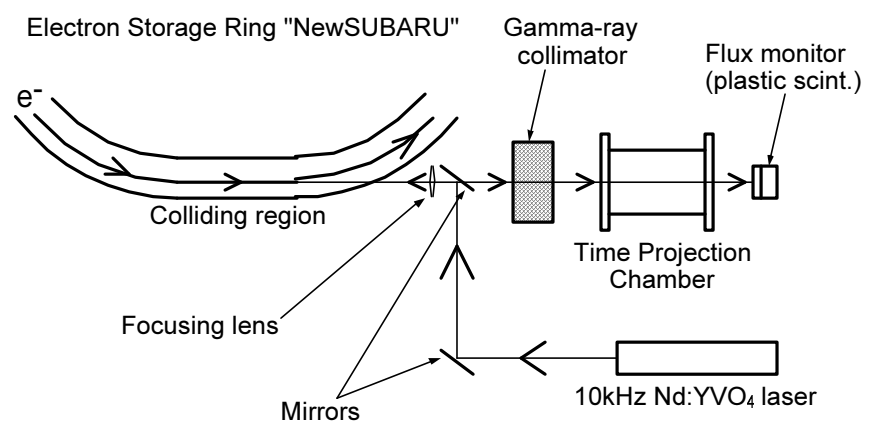

Figure 1: Schematic view of the experimental setup at the NewSUBARU LCS facility.

impinged upon the gas target contained in a time-projection chamber (TPC) [22]. Since the gas target was a mixture of natural helium (80\%) and deuterated methane $\left(\mathrm{CD}_{4}, 20 \%\right)$, it served as an active target, which enabled us to detect the tracks and the energy losses of the charged particles emitted from the photonuclear reactions with the efficiency of nearly \%100the solid angle of $4 \pi$. The effective thickness, total pressure and the average temperature of the target gas were $210 \mathrm{~mm}$, $954.9 \pm 1.5$ Torr and $26.9 \pm 0.3{ }^{\circ} \mathrm{C}$, respectively. The $\gamma$-ray intensity was measured in real-time by counting the $\gamma$-rays traveling through the TPC using a plastic scintillation counter with a front $\mathrm{e}^{+} \mathrm{e}^{-}$ pair converter made of $2 \mathrm{~mm}$ thick lead sheet. The detection efficiency of the plastic scintillator was calibrated against a BGO counter whose dimension and absolute efficiency were $50.8 \mathrm{~mm} \phi$ $\times 152.4 \mathrm{~mm}$ and better than $99.5 \%$, respectively. The energy distribution of the LCS $\gamma$-rays was measured with a $\mathrm{NaI}(\mathrm{Tl})$ detector with the size of $152.4 \mathrm{~mm} \phi \times 127.0 \mathrm{~mm}$.

Using the electron beam with the energy of $0.974 \sim 1.46 \mathrm{GeV}$ and the $10 \mathrm{kHz}$ pulsed $\mathrm{Nd}: \mathrm{YVO}_{4}$ laser with the fundamental $(1064 \mathrm{~nm})$ and second harmonic $(532 \mathrm{~nm})$ waves, the $\gamma$-rays with the maximum energies ranging from $16.7 \mathrm{MeV}$ to $37.2 \mathrm{MeV}$ were generated. The total $\gamma$-ray intensity is typically $2 \times 10^{4}$ photons/s at the average $\gamma$-ray energy of $E_{\gamma}=32 \mathrm{MeV}$ with the laser power of $1 \mathrm{~W}$ and an stored electron beam current of $200 \mathrm{~mA}$ [23].

The data were acquired event by event using a data-acquisition system made of the standard NIM and CAMAC circuits. The trigger rate and the dead time of the data acquisition were typically $30 \mathrm{~Hz}$ and $10 \%$, respectively.

\section{Photodisintegration of ${ }^{4} \mathrm{He}$}

The true events of the photonuclear reactions of ${ }^{4} \mathrm{He}$ were clearly observed with the TPC as demonstrated by the examples of the particle tracks shown in Fig. 2.

The ${ }^{4} \mathrm{He}(\gamma, \mathrm{p})^{3} \mathrm{H},{ }^{4} \mathrm{He}(\gamma, \mathrm{n}){ }^{3} \mathrm{He}$ and ${ }^{4} \mathrm{He}(\gamma, \mathrm{pn})^{2} \mathrm{H}$ reaction events were identified according to the numbers and the angles of the tracks and the total energy losses. The cross sections of the ${ }^{4} \mathrm{He}(\gamma, \mathrm{p}){ }^{3} \mathrm{H},{ }^{4} \mathrm{He}(\gamma, \mathrm{n}){ }^{3} \mathrm{He}$ and ${ }^{4} \mathrm{He}(\gamma, \mathrm{pn})^{2} \mathrm{H}$ reactions were deduced from the yields of those reaction events, target thickness and the incident $\gamma$-ray flux. The detection efficiency of the TPC for each reaction channels was determined by comparing the measured energy loss spectrum and that of the Monte Carlo simulation. Fig. 3 shows the measured and calculated energy loss spectra for 


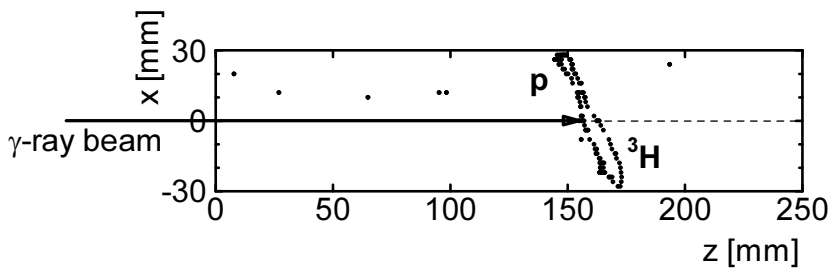

(a)

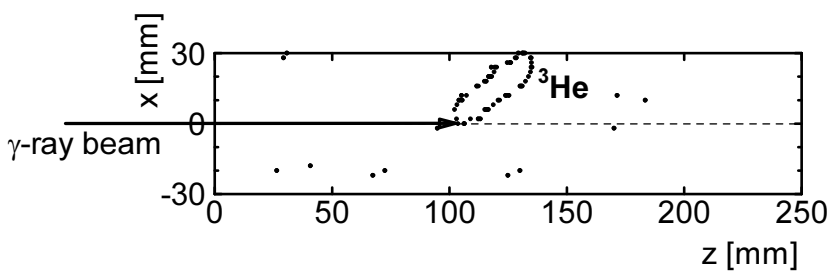

(b)

Figure 2: Examples of the observed tracks of the charged particles emitted by the photonuclear reactions of ${ }^{4} \mathrm{He}:(\mathrm{a}){ }^{4} \mathrm{He}(\gamma, \mathrm{p})^{3} \mathrm{H}$ and (b) ${ }^{4} \mathrm{He}(\gamma, \mathrm{n}){ }^{3} \mathrm{He}$. The boxes indicate the border of the effective region of the TPC. The $\gamma$-rays entered the effective region from the left side. The tracks are indicated by the dots corresponding to the drift times of the leading and trailing edges of the signals detected by the cathode wires of the TPC.
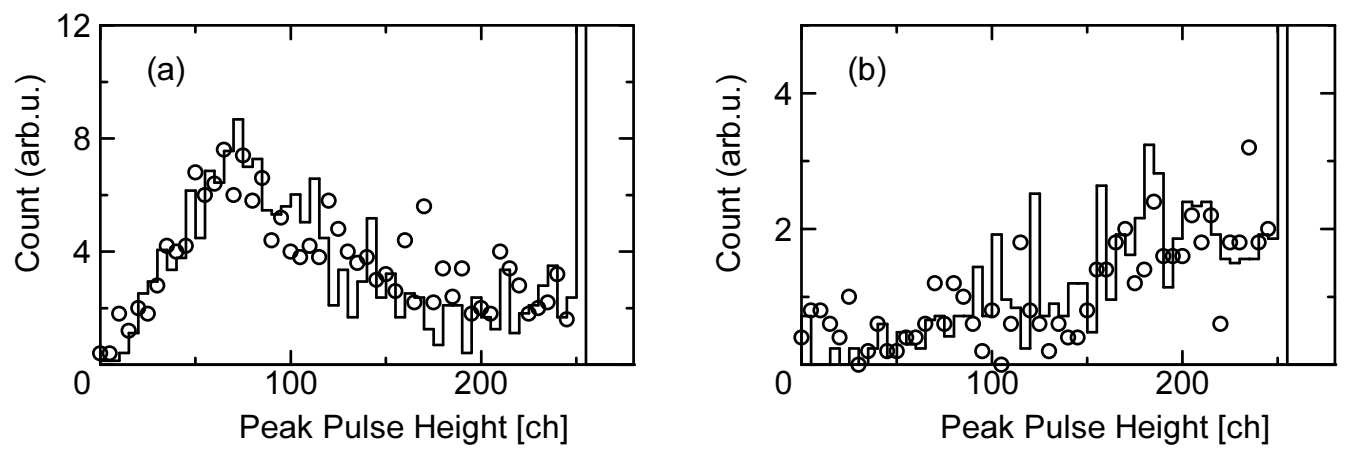

Figure 3: Energy loss spectra of the charged particles emitted by the photonuclear reactions of ${ }^{4} \mathrm{He}$ : (a) ${ }^{4} \mathrm{He}(\gamma, \mathrm{p})^{3} \mathrm{H}$ and (b) ${ }^{4} \mathrm{He}(\gamma, \mathrm{n})^{3} \mathrm{He}$. Open circles and histograms indicate the experimental data and the Monte Carlo calculations, respectively. The peaks at 250 channel stand for the saturation in the analog-to-digital converter.

the ${ }^{4} \mathrm{He}(\gamma, \mathrm{p})^{3} \mathrm{H}$ and ${ }^{4} \mathrm{He}(\gamma, \mathrm{n})^{3} \mathrm{He}$ reactions. Since the threshold level for the analog signal of the TPC was set at 7.0 channel, the detection efficiency was determined to be $98.5 \pm 1.5 \%$. for both the ${ }^{4} \mathrm{He}(\gamma, \mathrm{p})^{3} \mathrm{H}$ and ${ }^{4} \mathrm{He}(\gamma, \mathrm{n}){ }^{3} \mathrm{He}$ reactions. Fig. 4 shows the preliminary result on the cross sections of the ${ }^{4} \mathrm{He}(\gamma, \mathrm{p})^{3} \mathrm{H}$ and ${ }^{4} \mathrm{He}(\gamma, \mathrm{n})^{3} \mathrm{He}$ reactions obtained from the present measurement.

\section{Photodisintegration of the deuteron}

In the present experiment, we used a gas target containing $\mathrm{CD}_{4}$ in order to study the accuracy of the measurement by detecting the photodisintegration of the deuteron, whose cross section has been well known both experimentally and theoretically. As shown in Fig. 5, the preliminary cross sections from the present measurement are in a good agreement with the existing data, supporting 

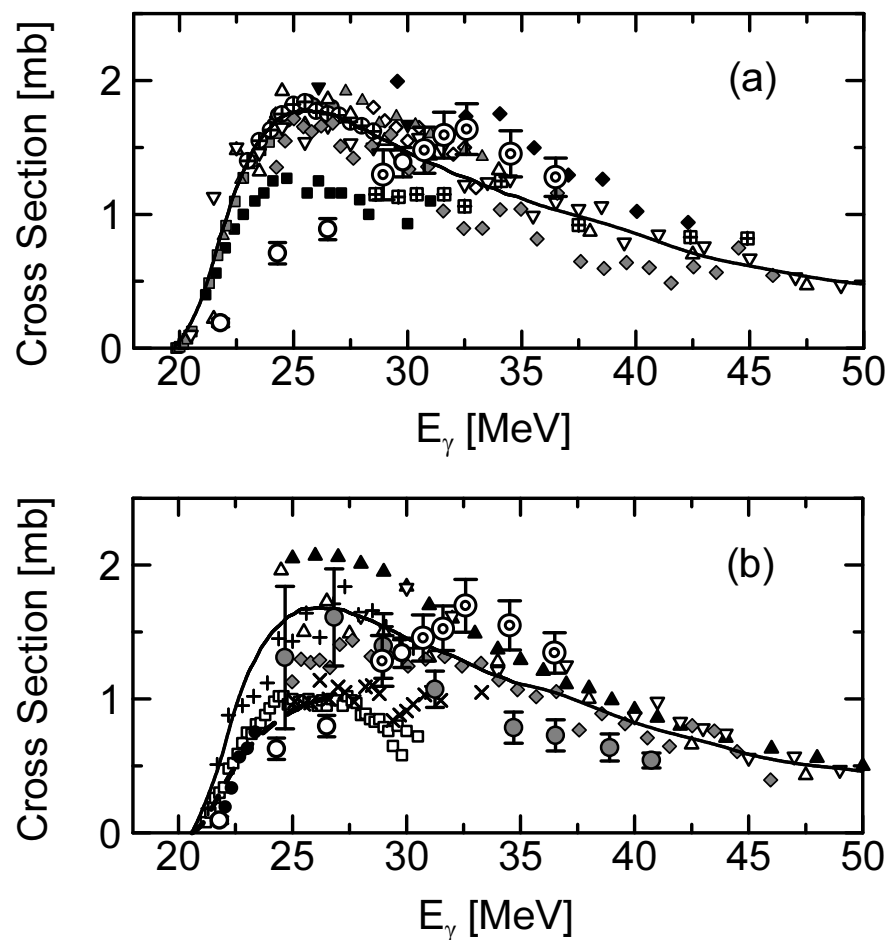

Figure 4: Cross sections of the photonuclear reactions of ${ }^{4} \mathrm{He}$ (preliminary): (a) ${ }^{4} \mathrm{He}(\gamma, \mathrm{p})^{3} \mathrm{H}$ and (b) ${ }^{4} \mathrm{He}(\gamma, \mathrm{n})^{3} \mathrm{He}$. The open circles, the double circles, and the gray circles denote our previous data measured at AIST [20], the present work, and the cross sections from Ref. [24, 25], respectively. The other symbols indicate the previous data (see the references in Ref. [20]). The solid curves and the dashed curve denote theoretically calculated cross sections from Ref. [5] and Ref. [3], respectively.

the accuracy of the present method in the determination of the photonuclear reaction cross sections.

\section{Summary}

The cross sections of the photonuclear reactions of ${ }^{4} \mathrm{He}$ were measured up to the maximum $\gamma$-ray energy of $37.2 \mathrm{MeV}$ using the NewSUBARU LCS $\gamma$-ray beam and a time-projection chamber with an active gas target. The reliability of the present method was studied by simultaneously measuring the photodisintegration of the deuteron whose cross section has been well known. The present result on the photodisintegration of ${ }^{4} \mathrm{He}$ was consistent with our previous one measured at TERAS in the overlapping energy region of $E_{\gamma}=29 \sim 31 \mathrm{MeV}$. The preliminary result showed the ${ }^{4} \mathrm{He}(\gamma, \mathrm{p})^{3} \mathrm{H}$ and ${ }^{4} \mathrm{He}(\gamma, \mathrm{n})^{3} \mathrm{He}$ reaction cross sections become maximum at around $E_{\gamma}=32 \sim 33$ $\mathrm{MeV}$, being different from many of other experimental data as well as the recent result of the theoretical calculation with the realistic potentials for the nucleon-nucleon and three-nucleon interactions [5]. On the other hand, the calculated $(\gamma, n)$ cross section with the Alt-Grassberger-Sandhas method [3] shows the similar energy dependence with the present one up to the upper end of the energy region in the calculation, and it will be important to extend the calculation up to $E_{\gamma} \sim 40$ $\mathrm{MeV}$. It is also interesting to measure the $\mathrm{E} 1$ photodisintegration cross sections in the higher energy 


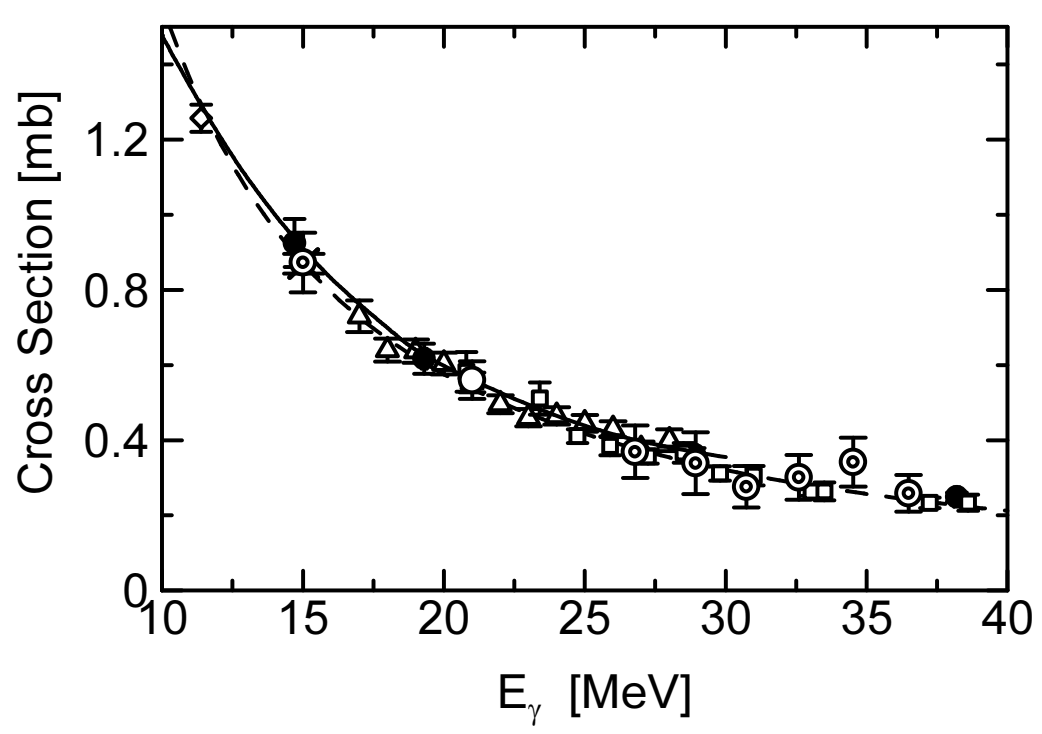

Figure 5: Photodisintegration cross section of the deuteron. The open circles and the double circles stand for our previous data [20] and the present work (preliminary). The other symbols indicate the data from Ref. [26] (filled circles), Ref. [27] (open squares), Ref. [28] (open triangles), Ref. [29] (open diamonds), and Ref. [30] (crosses). The solid curve and the dashed curve are the evaluated data [31] and the theoretical calculation with the momentum-space approach [32], respectively.

region using the linearly-polarized LCS $\gamma$-rays in order to survey the whole structure of the excitation functions and to obtain the E1 sum rules, which can be used for a model-independent test of the absolute strength of the E1 excitation. For that purpose an upgrade plan for the NewSUBARU LCS $\gamma$-ray source with an extended energy range is now under consideration.

\section{Acknowledgments}

The authors would like to thank Prof. T. Kajino for fruitful discussions. This work was supported in part by Grant-in-Aid for Specially Promoted Research of the Japan Ministry of Education, Science, Sports and Culture and in part by Grant-in-Aid for Scientific Research of the Japan.

\section{References}

[1] G. Ellerkmann, W. Sandhas, S. A. Sofianos, and H. Fiedeldey, Phys. Rev. C53 (1996) 2638.

[2] V.D. Efros, W. Leidemann, and G. Orlandini, Phys. Rev. Lett. 78 (1997) 4015.

[3] W. Sandhas et al., Nucl. Phys. A631 (1998) 210c.

[4] N. Barnea, V.D. Efros, W. Leidemann, and G. Orlandini, Phys. Rev. C63 (2001) 057002.

[5] S. Quaglioni et al., Phys. Rev. C69 (2004) 044002. 
[6] M. Sakai, Prog. Theor. Phys. 63 (1980) 180.

[7] D. Gazit et al., Phys. Rev. Lett. 96 (2006) 112301.

[8] S. Bacca, Phys. Rev. C75 (2007) 044001.

[9] S. Quaglioni and P. Navrátil, Phys. Lett. B652 (2007) 370.

[10] D. Halderson and R.J. Philpott, Phys. Rev. C28 (1983) 1000.

[11] D. Halderson, Phys. Rev. C70 (2004) 034607.

[12] T. Suzuki et al., Phys. Rev. C74 (2006) 034307.

[13] D. Gazit and N. Barnea, Phys. Rev. Lett. 98 (2007) 192501.

[14] S.E. Woosley, D.H. Hartmann, R.D. Hoffman, and W.C. Haxton, Astrophys. J. 356 (1990) 272.

[15] N. Ohnishi, K. Kotake, and S. Yamada, Astrophys. J. 667 (2007) 375.

[16] B.S. Meyer, Astrophys. J. 449 (1995) L55.

[17] T. Yoshida, M. Terasawa, T. Kajino, and K. Sumiyoshi, Astrophys. J. 600 (2004) 204.

[18] A. Heger et al., Phys. Lett. B606 (2005) 258.

[19] M. Kusakabe et al., Phys. Rev. D79 (2009) 123513.

[20] T. Shima et al., Phys. Rev. C72 (2005) 044004.

[21] K. Aoki et al., Nucl. Instr. Meth. in Phys. Res. A516 (2004) 228.

[22] T. Kii, T. Shima, T. Baba, and Y. Nagai, Nucl. Instr. Meth. in Phys. Res. A552 (2005) 329.

[23] S. Miyamoto et al., Radiation Meas. 41 (2006) S179-S185.

[24] B. Nilsson et al., Phys. Lett. B626 (2005) 65.

[25] B. Nilsson et al., Phys. Rev. C75 (2007) 014007.

[26] R. Bernabei et al., Phys. Rev. Lett. 57 (1986) 1542.

[27] C. Dupon et al., Nucl. Phys. A445 (1985) 13.

[28] D.M. Skopik, Y.M. Shin, M.C. Phenneger, and J.J. Murphy, II, Phys. Rev. C9 (1974) 531.

[29] Y. Birenbaum et al., Phys. Rev. C32 (1985) 1825.

[30] P. Michel, K. Moeller, J. Moesner, and G. Schmit, J. Phys. G15 (1989) 15.

[31] ENDF/B-VII data file for ${ }^{1} \mathrm{H}(\mathrm{MAT}=125)$. 2006, evaluated by G.M. Hale.

[32] H. Arenhövel and M. Sanzone, Few-Body Systems Suppl. 3: PhotodisintegrationoftheDeuteron (Springer-Verlag, Wien,1991). 